\title{
Development of Model to Identify the Factors for Risky Driving Behaviour by Analyzing EEG Waveform
}

\author{
Ashwini B. Sonulkar ${ }^{1}$, Sanjay S. Wankhede ${ }^{2}$ \\ ${ }^{1}$ P.G. Scholar, Department of Electronics, G. H. Raisoni College of Engineering, Nagpur, India \\ ${ }^{2}$ Professor, Department of Electronics, G. H. Raisoni College of Engineering, Nagpur, India
}

\begin{abstract}
Traffic accidents are caused due to the main three parameters as traffic environment, driver and vehicle. As driving is a complex behavior that can be affected by an individual psychological state, emotions and environment. For safe driving and eliminating risky driving of an individual it has become necessary for estimation of psychological state of the driver. In this method, the mental status of driver is measured by EEG Waves by recording electrical activity of the brain. By using EEG headset that senses the electrical signal and transmits to the computer wirelessly through Bluetooth which reduces the number of sensor and eliminated the use of liquid on scalp while measuring EEG waves. The extracted brainwaves are then used to estimate different brain features like attention, distraction and eye blinking. The brainwaves are trained by backpropagation neural network. And whenever the risky situation occurs or when driver seems to be distracted from driving, the alarm blows. It helps a lot to eliminate the risky situation ensures safe driving.
\end{abstract}

Keywords: EEG, driving risk, safe driving, backpropagation

\section{Introduction}

There are various types of accidents that may cause damage or death. The difficulties arising due to the damages and death caused due to the road accidents in India, grabs the attention of an individual to reduce the road accidents. Environment, vehicle and driver are some of the major parameters which affect the driving behavior [1]. Driver performance is the ability of what the driver can do relating to the skills, knowledge and mental ability and the driver behavior is what the driver chooses to do with its qualities. Among the parameters affecting driving behavior the psychological state of the driver is main factor. The neuropsychological attempt is useful to recognize the complex association of the person's mental situation that can cause risky driving acts. The different human state of mind is alert, distraction, drowsy, anxiety etc. must be known while driving so that whenever the driver's mind state swings from alert state it should be notified so that the risky driving behavior can be avoided.

The neuropsychological approach depends on brain wave indexes from which the mind state of a person can be identified so that decision regarding the ability of driver to drive can be made possible.

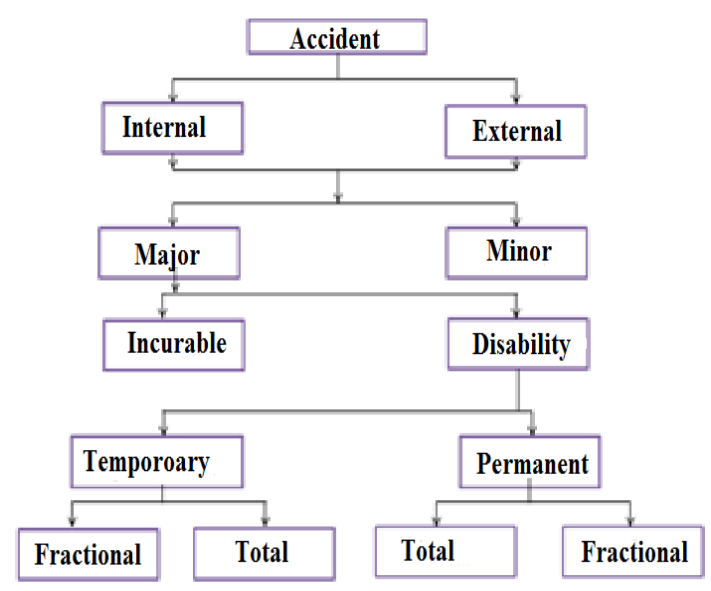

Figure 1: Types of accidents

\subsection{EEG}

The human brain consists of billions of intertwined neurons which communicate with each other and generate electricity. The activity created by these neurons is aggregated into waves which are measurable. EEG is an electrophysiological recording method to record electrical agility of the brain. It is typically noninvasive with the electrode positioned along the skull. Within the neurons of brain the voltage undulating resulting from the ionic currents is measured by EEG [3]. The different mind state of an individual can be detected by EEG brainwaves. Frequencies of the EEG brainwave define the different mind state of a person. These EEG brainwaves can be extracted with the help of hardware EEG headset.

EEG headset is a hardware which communicates with computer wirelessly. It measures the electrical pulses that are brainwave (alpha wave, beta wave, delta wave and theta 


\section{International Journal of Science and Research (IJSR) \\ ISSN (Online): 2319-7064 \\ Index Copernicus Value (2013): 6.14 | Impact Factor (2015): 6.391}

wave) with sensor and passes to the computer through Bluetooth.

\subsection{Brainwaves Types}

Depending on the amplitude and frequency the brainwaves are categorized into alpha, beta, theta and delta waves. When the mind state is awakened and enthusiastically involved in intellectual events, produces beta waves. This wave is comparatively more active as compare to the other brainwaves and its frequency range is 14-40 cycles per second. Beta waves have features of an intensely engaged mind this wave characterizes awakening. In case of frequency the next category of brainwave is alpha. This wave characterizes non- awakening and are higher in amplitude. Frequency range of alpha wave is from 8-13 cycles per second. Theta brainwave is typically of even maximum amplitude and low frequency and the frequency range of this brainwave is between 4-7 cycles per second. Delta brainwaves have slowest frequency and maximum amplitude. The frequency typically having a range of 0.5-3 cycles-persecond. It does not tend to zero because that would cause "brain dead" [2].

\begin{tabular}{|c|c|c|}
\hline $\begin{array}{c}\text { Brainwave } \\
\text { Types }\end{array}$ & $\begin{array}{c}\text { Frequency } \\
\text { range }\end{array}$ & Mental states and condition \\
\hline Theta & $4 \mathrm{~Hz}$ to $7 \mathrm{~Hz}$ & $\begin{array}{c}\text { Intuitive, creative, recall, } \\
\text { fantasy, imaginary, dream. }\end{array}$ \\
\hline Alpha & $8 \mathrm{~Hz}$ to $12 \mathrm{~Hz}$ & $\begin{array}{c}\text { Relaxed(but not drowsy) } \\
\text { tranquil, conscious }\end{array}$ \\
\hline Low Beta & $12 \mathrm{~Hz}$ to $15 \mathrm{~Hz}$ & $\begin{array}{c}\text { Formerly SMR, relaxed yet } \\
\text { focused, Integrated }\end{array}$ \\
\hline Midrange Beta & $16 \mathrm{~Hz}$ to $20 \mathrm{~Hz}$ & $\begin{array}{c}\text { Thinking, aware of self and } \\
\text { surroundings }\end{array}$ \\
\hline High Beta & $21 \mathrm{~Hz}$ to $30 \mathrm{~Hz}$ & Alertness, agitation \\
\hline
\end{tabular}

Figure 2: EEG waves decomposition according to brain state

\section{Methodology}

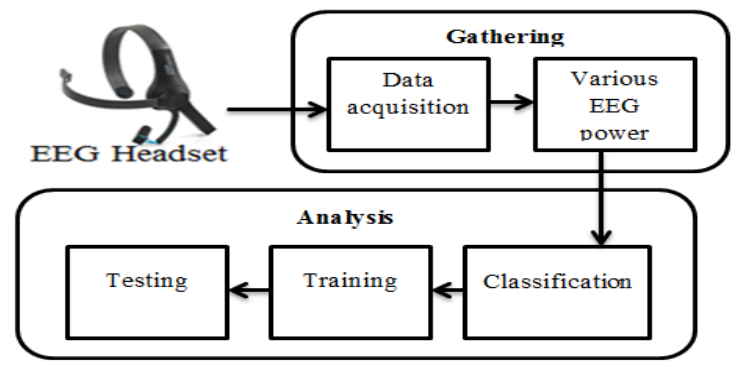

Figure 3: Block Diagram

For detecting human cognitive state, focusing on the arenas to measure psychological variations such as eye blinking, pulse rate, heart rate, skin electric potential and brain waves. The human electroencephalographic (EEG) consist of abundant information of the mental states such as alertness, distraction, anxiety, happiness etc. is revealed by many researchers. But relatively less information has been retrieved till the signal processing methods are capable of extracting enough to process the data in real time. These methodologies depending on EEG waveforms have the benefits for creating precise and quantitative judgments of psychological states by hardware EEG headset and software set up. The headset is connected serially to transmit the EEG waves to computer through Bluetooth. By analyzing the EEG waves the mental state of an object can be estimated and is displayed on command window.

The back propagation neural network is learning method which is used to train the database created by the EEG brainwave. Due to which the accurate and continuous fluctuation in mind state can be predicted. It is a supervised learning method which helps in minimizing error. Backpropagation neural network consist of three layers as input layer, hidden layer, output layer. The network receives input from neurons on input layer and output from neurons on output and then error is calculated. The error is then backpropagated from output to input so that weights are updated and error can be minimized for better training pattern.

net $_{\mathrm{j}}=\sum_{\mathrm{i}} \mathrm{W}_{\mathrm{ij}} \mathrm{O}_{\mathrm{i}}+\Theta_{\mathrm{j}}$ net $_{\mathrm{k}}=\sum_{\mathrm{j}} \mathrm{W}_{\mathrm{kj}} \mathrm{O}_{\mathrm{j}}+\Theta_{\mathrm{k}}$

Where $\mathrm{i}$ is input layer, $\mathrm{j}$ is hidden layer, $\mathrm{k}$ is output layer, $\Theta_{j}$ and $\Theta_{k}$ are errors in hidden layer and output layer respectively. $\mathrm{W}_{\mathrm{ji}}$ are weight update in between input layer and hidden layer and $\mathrm{W}_{\mathrm{kj}}$ is weight update in between hidden layer and output layer.

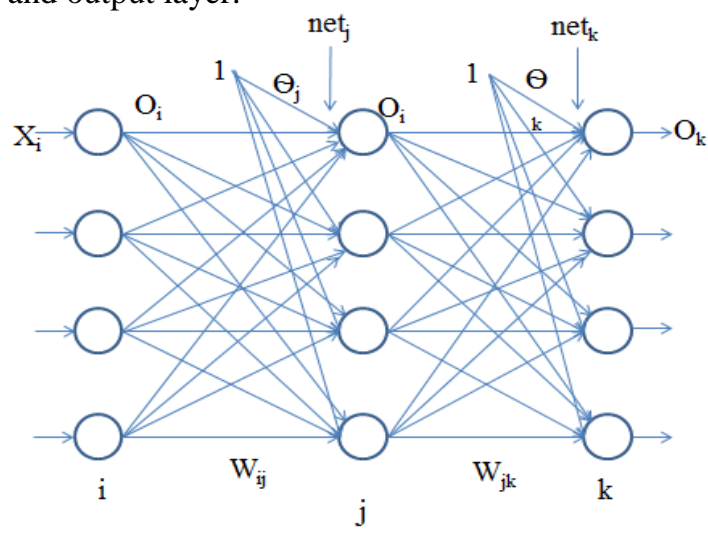

Figure 4: backpropagation network layer

Output of neuron $\mathrm{j}$ is :

$\mathrm{O}_{\mathrm{j}}=\mathrm{f}\left(\right.$ net $\left._{\mathrm{j}}\right)=\frac{1}{1+(\varepsilon)-\text { net }}$

Output of neuron $\mathrm{k}$ is:

$\mathrm{O}_{\mathrm{k}}=\mathrm{f}\left(\right.$ net $\left._{\mathrm{k}}\right)=\frac{1}{1+(e)^{- \text {netk }}}$

\section{Experimental Set Up}

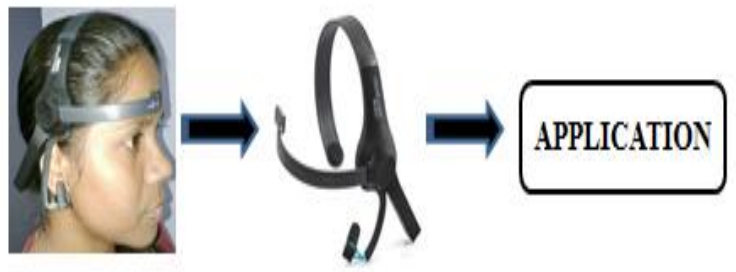

Figure 5: Experimental set up

While driving, traffic events are related to the human cognitive state. Distraction (nervousness \& anger) alertness 


\section{International Journal of Science and Research (IJSR) \\ ISSN (Online): 2319-7064}

Index Copernicus Value (2013): 6.14 $\mid$ Impact Factor (2015): 6.391

and eye blinking all are related with driver ability, safety and risk bearing. Consequences for high eye blinking and distraction are higher possibilities for negative events that is, negative activities effects on risk perception. There is a moderate relationship between the driving accidents and destructions, and drivers without driving destructions favored lower levels of risk-taking behavior, and the personality traits of those who displayed more pleasure in organizing and anticipating goals rather than instantaneous sensations, and expressed a higher need for individual control, were significantly less risk-taking in their behavior.

The headset is connected to five different subjects in different timings and data is being collected for different mental state for different driving environment. The set up simply comprises of a headset, an ear-clip, and a sensor. The headset ground and reference electrodes are placed on the ear clip, whereas EEG electrode is on sensor arm, resting on the forehead of person.

The signals are passed serially to the computer. Comport and the baudrate of the sensor and computer must be set for serial transmission of signal. After this the data base of different object at different time is collected. The collected data base is then trained by backpropagation neural network.

After training, database has to be tested in which the features of a brainwave are extracted in terms of frequency versus amplitude. For the features extraction the extracted brainwave is passed through low pass filter and high pass filter with the following function:

test feature=features (ndat);

$a=\overline{s i m}$ (net, test_feature);

In testing database, the similarities in features of brainwave are examined, and are denoted according to the brainstate that is alert state, distracted state or eye blink state.

Finally for the real time experimentation the live brainwaves and are compared with the trained waveforms and the features of the brainwave which best relates to live waveform is compared and accordingly brainstate of a person is displayed. Whenever the person's state of mind is distracted, the alarm blows to alert the person for safe driving.

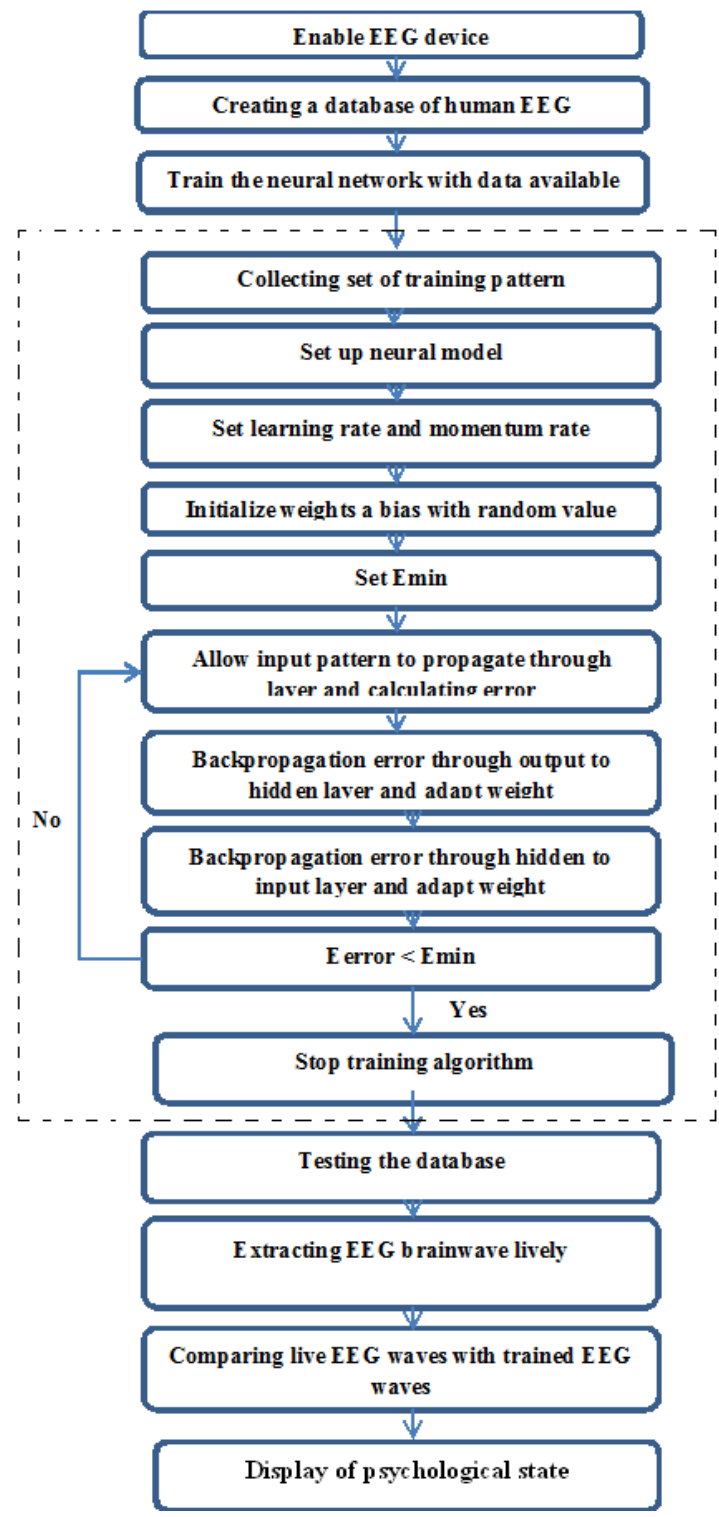

Figure 6: Flow chart for detecting mind state

Table 1: Extracted features of brainwave of attention and distraction

\begin{tabular}{|c|c|c|c|}
\hline & & Attention(mV) & Distraction $(\mathrm{mV})$ \\
\hline \multirow{4}{*}{ Max } & 1 & 87 & 249 \\
\cline { 2 - 4 } & 2 & 79 & 281 \\
\cline { 2 - 4 } & 3 & 81 & 264 \\
\cline { 2 - 4 } & 4 & 74 & 294 \\
\cline { 2 - 4 } & 5 & 98 & 224 \\
\hline \multirow{5}{*}{$\begin{array}{c}\text { Band } \\
\text { power }\end{array}$} & 1 & 2408.8922 & 30266.0626 \\
\cline { 2 - 4 } & 2 & 2757.1253 & 33388.2255 \\
\cline { 2 - 4 } & 3 & 2507.9273 & 32349.6165 \\
\cline { 2 - 4 } & 4 & 2508.9624 & 34724.8847 \\
\hline \multirow{5}{*}{ Standard } & 5 & 3188.1453 & 25823.0676 \\
\cline { 2 - 4 } & 1 & 20.2787 & 63.5570 \\
\cline { 2 - 4 } & 2 & 18.3921 & 69.7178 \\
\cline { 2 - 4 } & 3 & 17.9394 & 67.2718 \\
\hline & 4 & 17.0176 & 72.7278 \\
\hline \multirow{4}{*}{ Variance } & 5 & 21.2682 & 54.8558 \\
\cline { 2 - 4 } & 1 & 411.2278 & 4039.4944 \\
\cline { 2 - 4 } & 2 & 338.2701 & 4860.5814 \\
\cline { 2 - 4 } & 3 & 321.8237 & 4525.5068 \\
\hline
\end{tabular}

Volume 5 Issue 6, June 2016 www.ijsr.net 


\section{International Journal of Science and Research (IJSR) \\ ISSN (Online): 2319-7064 \\ Index Copernicus Value (2013): 6.14 | Impact Factor (2015): 6.391}

\section{Results}

The developed Application retrieves various information and features of brainwave describing the mental state and ability of a person to drive safely.
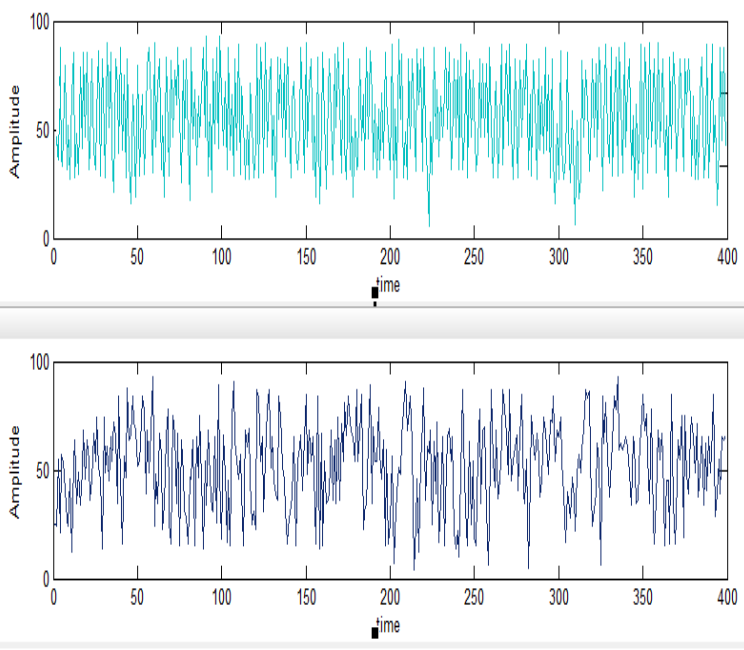

Figure 7: EEG brainwave

The figure 7 shows a human EEG brainwave time versus amplitude the light blue colored waveform shows distracted brainwave and the dark blue colored brainwave shows attention brainwave .

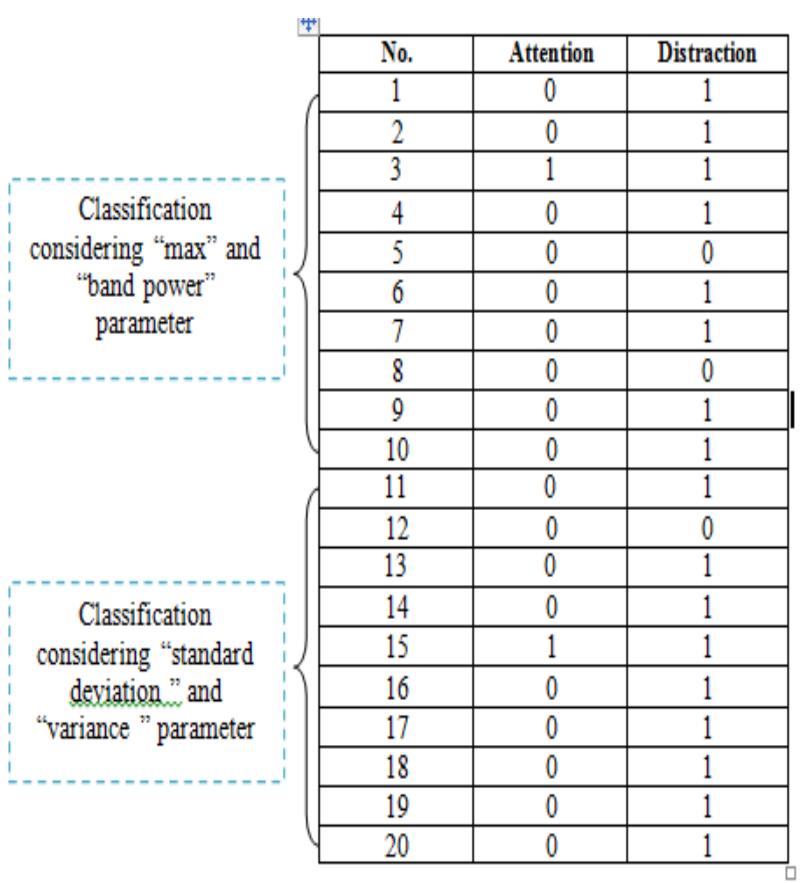

Figure 8: Classification result of database

In this the database is created of different psychological state and the database is trained with the help of backpropagation neural network. In the above result the database is trained and according to the brainwaves the status is displayed.

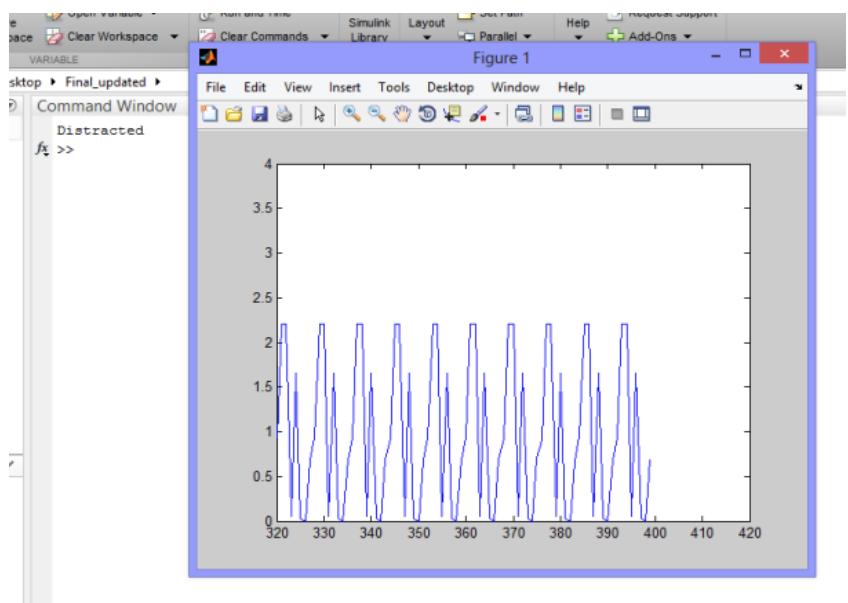

Figure 9: live test

In figure 9 after training the brainwaves the live test has been taken and as per the brainwave type the status is displayed in command window that is the distracted brainwave is displayed.

\section{Conclusion}

The experiment results describe the person's mental status. These results can be further reused for safety driving as whenever there is arrival of a risky situation or EEG waves shows the mental disability of a person to drive, the system will blow an alarm in order to alert the person.

\section{References}

[1] Rohit Mankar\#1, Pranav Bawane\#2, Tushar Landge\#3 Palash Dalal\#4,Vivek Gour\#5 "Brain Wave Sensing”. 2014 Annual IEEE India Conference (INDICON)

[2] Mikio DANNO, Member, IEEE, and Akio WAKABAYASHI "Neuropsychological Approach to Identifying Risky Driving Behaviors" 2010 IEEE Intelligent Vehicles Symposium University of California, San Diego, CA, USA June 21-24,2010

[3] Chin-Teng Lin*, Yu-Chieh Chen, Ruei-Cheng Wu, Sheng-Fu Liang, and Teng-Yi Huang Brain Research Center "Assessment of Driver's Driving Performance and Alertness Using EEG-based Fuzzy Neural Networks" Brain Research Center IEEE 2005

[4] Dibakar Barua, Pranshu Jain, Jitesh Gupta, Dhananjay V. Gadre Dept. of Electronics and Communication " Road accident prevention unit (r.a.p.u)" 2013 Texas Instruments India Educators' Conference.

[5] Anup Doshi and Mohan M. Trivedi "Tactical Driver Behavior Prediction and Intent Inference: A Review" 14th International IEEE 2011 Conference on Intelligent Transportation Systems

[6] Ruei-Cheng Wu*, Chin-Teng Lin, Sheng-Fu Liang, Te-Yi Huang, and Yu-Chieh Chen " Estimating Driving Performance Based on EEG Spectrum and Fuzzy Neural Network" IEEE 2004.

[7] Sivachitra.M and Vijayachitra.S "Planning and Relaxed state EEG signal classification using Complex Valued Neural Classifier for Brain Computer Interface" IEEE 2015 
[8] Muhammad bin Yahya, Zunairah $\mathrm{Hj}$ Murat, Afiqah Qumira Binti Ismail "Temporal Hemispheric Dominance of Omega-3: Measurement of Alpha and Beta Wave Signals Using EEG" 2013 International Conference on Electrical, Electronics and System Engineering

[9] Denis Renato de Moraes Piazentin and Joao Luis Garcia Rosa, Member, IEEE "Motor imagery classification for Brain-Computer Interfaces through a chaotic neural network" 2014 International Joint Conference on Neural Networks (IJCNN)

Volume 5 Issue 6, June 2016 www.ijsr.net 